\title{
Postmortem evaluation of cholesterol, triglyceride, and apolipoprotein levels
}

\author{
Charlotte Girard $^{1} \cdot$ Maria Pia Scarpelli $^{1}$ - Camilla Tettamanti ${ }^{2} \cdot$ Cristian Palmiere $^{1}$ (D)
}

Received: 1 June 2017 / Accepted: 11 August 2017 / Published online: 18 August 2017

(C) Springer-Verlag GmbH Germany 2017

\begin{abstract}
Cholesterol and triglyceride levels have been analyzed in the forensic setting and their values correlated with atherosclerotic lesions found at autopsy and histology. Nevertheless, the results of these investigations have provided diverging information on postmortem molecule stability and postmortem measurement reliability. The aim of this study was to determine triglycerides, total cholesterol, low-density and high-density lipoprotein cholesterol, apolipoprotein $\mathrm{B}_{100}$, and apolipoprotein A-I in antemortem and postmortem serum samples in a series of cases $(N=10$, including cardiac and noncardiac deaths) that underwent forensic investigations and had both samples available, measure the same molecules in postmortem serum from femoral blood and pericardial and pleural fluids $(N=39$, including cardiac and noncardiac deaths), and evaluate whether different levels of these molecules could be observed in cases characterized by different degrees of coronary artery atherosclerosis $(N=39$, including cardiac and noncardiac deaths). Preliminary results indicated that total cholesterol and low-density and high-density lipoprotein cholesterol levels in postmortem serum samples tended to be lower than those in antemortem specimens, whereas triglyceride levels in postmortem serum samples tended to be higher than those in antemortem samples. No relationship could be found between postmortem serum and pericardial fluid levels or between postmortem serum and pleural fluid levels of all tested biomarkers. Lastly, cases
\end{abstract}

Cristian Palmiere

cristian.palmiere@chuv.ch

1 CURML, University Center of Legal Medicine, Lausanne University Hospital, Chemin de la Vulliette 4, 1000 Lausanne, Switzerland

2 Department of Legal Medicine, University of Genova, Via de Toni 12 , Genoa, Italy characterized by severe coronary artery atherosclerosis revealed higher postmortem serum levels of total cholesterol and apolipoprotein B. Globally considered, these data confirm that femoral blood postmortem serum levels of cholesterol and apolipoproteins may be considered suitable to estimate their antemortem values in forensic cases characterized by coronary artery atherosclerosis.

Keywords Postmortem biochemistry $\cdot$ Autopsy . Cholesterol · Triglycerides

\section{Introduction}

Atherosclerotic cardiovascular disease, including coronary artery disease, remains a leading cause of death and disability in most Western countries. Among the various risk factors, numerous clinical studies have demonstrated a strong association between hypercholesterolemia and coronary heart disease. However, more recent research has provided evidence for triglycerides to be treated as an independent risk factor for atherosclerotic cardiovascular disease [1-8].

Forensic pathologists routinely encounter autopsies for sudden coronary death in young adults and can be aided by recent advances in postmortem imaging techniques and protocols. These now allow the extent of coronary atherosclerosis to be estimated with precision. Indeed, correlations between cholesterol/triglyceride profiles and atherosclerotic lesions would significantly contribute to better understanding the pathogenesis of death, especially in those cases where antemortem data on blood lipids are unavailable. On the other hand, identification of elevated cholesterol levels in younger individuals with severe coronary artery disease may be a marker for genetic predisposition and thus deserves to be confirmed by further analysis. 
Cholesterol and triglyceride levels in antemortem and postmortem serum samples as well as pericardial fluid in both humans and animals have been investigated and compared in the past by some research teams. Furthermore, their values have been correlated with atherosclerotic lesions and coronary artery disease found at autopsy and histology. The results of these studies, though promising, have provided diverging information on postmortem molecule stability and postmortem measurement reliability. This has lead to the conclusion that lipid profile interpretation in postmortem samples best be tempered by several caveats [9-13].

The purpose of the study presented herein was threefold: (a) to determine triglycerides, total cholesterol, low-density lipoprotein cholesterol, high-density lipoprotein cholesterol, apolipoprotein $\mathrm{B}_{100}$, and apolipoprotein A-I in a series of cases that underwent postmortem investigations and for which antemortem and postmortem serum samples were available for comparison, thereby investigating the postmortem stability of these compounds, (b) to measure the same molecules in postmortem serum from femoral blood and pericardial and pleural fluids in a series of cases that underwent medicolegal investigations, thereby evaluating the distribution of these molecules in various biological fluids collected at autopsy and the usefulness of their determination in several biological samples for diagnostic purposes in the forensic setting, and (c) to evaluate whether different levels of these molecules could be observed in cases characterized by different degrees of coronary artery atherosclerosis at postmortem investigations.

\section{Materials and methods}

\section{Study design}

The present study was performed in 2016-2017 and was designed as a single-center study. All selected cases originated from forensic practice and underwent medicolegal autopsies as requested by local inquiring authorities (the public prosecutor). Laboratory analyses, including measurements of triglyceride, total cholesterol, low-density lipoprotein cholesterol, high-density lipoprotein cholesterol, apolipoprotein $\mathrm{B}_{100}$ (indicated as apolipoprotein B), and apolipoprotein A-I, were performed as part of the medicolegal investigations.

\section{Study populations}

To address the first purpose of the study, comparisons between antemortem and postmortem serum levels of triglycerides, total cholesterol, low-density lipoprotein cholesterol, highdensity lipoprotein cholesterol, apolipoprotein B, and apolipoprotein A-I were performed on samples collected from 10 individuals ( 8 males and 2 females, with a mean age of
48 years, range 39-69 years) who were admitted to hospital shortly prior to death.

Diagnosis on admission included sudden loss of consciousness in the presence of witnesses. Cardiopulmonary resuscitation attempts were performed on-site by co-workers, bystanders, or laypersons until paramedic arrival. Information pertaining to preexisting cardiovascular risk factors, including hypertriglyceridemia or hypercholesterolemia, was unavailable at hospital admission.

Antemortem samples consisted of serum samples obtained rapidly on admission to hospital. Death was pronounced in all cases within approximately $1 \mathrm{~h}$ of arrival.

Complete medicolegal autopsies were performed between 12 and $48 \mathrm{~h}$ after death. Postmortem samples consisted of serum samples obtained from peripheral (femoral blood) prior to autopsy.

Causes of death at postmortem investigations included cardiac deaths ( 7 cases) and drug intoxication (3 cases).

To address the second and third aims of the study, comparisons between postmortem serum and pericardial and pleural fluid levels of triglyceride, low-density lipoprotein cholesterol, high-density lipoprotein cholesterol, apolipoprotein B, and apolipoprotein A-I were performed on samples collected from 39 individuals (the 10 cases described earlier and 29 other cases admitted to the medicolegal center upon request of local inquiring authorities). The latter included 20 males and 9 females, with a mean age of 52 years (range $31-72$ years) with deaths occurring outside the hospital. Complete medicolegal autopsies were performed between 18 and $48 \mathrm{~h}$ after death. Causes of death included cardiac deaths (13 cases out of 29), hanging ( 6 cases out of 29), and drug intoxication (10 cases out of 29).

The 20 cardiac causes of death ( 7 from the first study group and 13 from the second study group) included:

1. Five cases macroscopically and microscopically characterized by various degrees of coronary artery atherosclerosis with evidence of fatty deposits in the vessel wall though without evidence of significant luminal narrowing or acute coronary thrombosis,

2. Five cases macroscopically and microscopically characterized by various degrees of coronary artery atherosclerosis and acute coronary thrombosis or rupture/erosion of a coronary atherosclerotic plaque,

3. Ten cases macroscopically and microscopically characterized by severe coronary artery atherosclerosis, with evidence of chronic occlusions of various degrees in the coronary artery lumen and foci of interstitial fibrosis within the myocardium without evidence of acute coronary thrombosis.

Case inclusion criteria for the first study group consisted of antemortem and postmortem serum availability as well as 
absence of intravenous fluid administration or minimal intravenous fluid administration.

Case inclusion criteria for the second study group consisted of postmortem serum as well as pericardial and pleural fluid availability at autopsy.

\section{Postmortem investigations and sample collection}

Conventional medicolegal autopsies, histology, toxicology, and biochemical investigations were performed in all cases. Conventional histology included hematoxylin-eosin (HE) stains of brain, heart, lung, liver, and kidney samples.

Systematic toxicological analysis included blood ethanol level determination as well as general screening for volatile and nonvolatile drugs, poisons, and metabolites.

Peripheral blood from the femoral veins was systematically collected for toxicological and biochemical studies prior to autopsy. Femoral blood samples were collected by aspiration with sterile needles and syringes from the femoral vein(s). Blood samples were drawn after clamping the vein(s) at the proximal end and keeping the lower limb(s) raised for several minutes. Samples were stored in tubes containing sodium fluoride and preservative-free gel serum separator tubes. The latter were centrifuged immediately post collection at $3000 \mathrm{~g}$ for $15 \mathrm{~min}$. After centrifugation, the separated supernatant (postmortem serum) was collected and stored in preservative-free tubes. No specimens were excluded due to insufficient sample volume. Postmortem serum samples were transferred to the laboratories immediately post collection. When analyses were delayed, samples were stored at $-20{ }^{\circ} \mathrm{C}$.

Undiluted samples of pericardial and pleural fluid were collected immediately post pericardium incision and pleural cavity opening during autopsy. All samples were immediately centrifuged at $3000 \mathrm{~g}$ for $15 \mathrm{~min}$. After centrifugation, the separated supernatants were collected and stored in preservativefree tubes. No specimens were excluded due to insufficient sample volume. The samples were transferred to the laboratories immediately post collection. When analyses were delayed, samples were stored at $-20^{\circ} \mathrm{C}$.

\section{Laboratory assays}

Cholesterol was determined by the CHOD-PAP (cholesterol oxidase phenol 4-aminoantipyrine peroxidase) method and triglycerides by the GPO-PAP (glycerol phosphate oxidase$p$-aminophenazone) on an automatic analyzer. Results were expressed in millimoles per liter. Apolipoprotein B and apolipoprotein A-I levels were determined in the selected fluids by immunonephelometric assay. Results were expressed in grams per liter.

For the purpose of this study, an increased level of triglycerides, low-density lipoprotein cholesterol, high-density lipoprotein cholesterol, apolipoprotein B, and apolipoprotein
A-I in postmortem serum from femoral blood was defined as that exceeding the highest upper limit of the clinical reference value indicated by the clinical laboratory where the analysis was performed. Cutoff values in pericardial and pleural fluids collected at autopsy were not preliminarily identified.

\section{Statistical analysis}

Comparisons of triglyceride, total cholesterol, low-density lipoprotein cholesterol, high-density lipoprotein cholesterol, apolipoprotein B, and apolipoprotein A-I levels in antemortem and postmortem serum samples were performed using the non-parametric Mann-Whitney $U$ test. Analogously, comparisons of triglyceride, total cholesterol, low-density lipoprotein cholesterol, high-density lipoprotein cholesterol, apolipoprotein B, and apolipoprotein A-I levels in postmortem serum and pericardial and pleural fluid were performed using the nonparametric Mann-Whitney $U$ test. The relationship among molecule concentrations in postmortem serum, pericardial fluid, and pleural fluid in each subgroup was also explored. Statistical significance was defined as a $p$ value of less than 0.05. All statistical analyses were performed using GraphPad Prism 4.0 (GraphPad Software, La Jolla, CA, USA).

\section{Results}

Concerning the first aim of our study, no statistically significant differences were noticed between antemortem and postmortem serum levels of total cholesterol, low-density lipoprotein cholesterol, high-density lipoprotein cholesterol, apolipoprotein B, and apolipoprotein A-I up to $48 \mathrm{~h}$ after death. Total cholesterol, low-density lipoprotein cholesterol, and highdensity lipoprotein cholesterol levels in postmortem serum samples tended to be lower than those in antemortem specimens. Nevertheless, none of the four individuals (out of the ten studied) with increased antemortem levels of these molecules had normal postmortem levels.

Conversely, triglyceride levels in postmortem serum samples tended to be higher than those in antemortem specimens, and differences between antemortem and postmortem serum concentrations were found to be statistically significant ( $p=0.039)$. Postmortem serum triglyceride levels were higher than the clinical reference values in four out of eight studied individuals that had normal antemortem serum triglyceride levels.

Concerning the second aim of our study, the differences among the concentrations of all measured molecules in all tested biological samples were found to be statistically significant. No relationship could be found between postmortem serum and pericardial fluid levels or between postmortem serum and pleural fluid levels of all tested biomarkers, irrespective coronary artery disease severity. 
Lastly, concerning the third aim of our study, postmortem cases characterized by severe coronary artery atherosclerosis and significant coronary artery luminal narrowing revealed higher postmortem serum levels of total cholesterol and apolipoprotein B than cases having minimal or insignificant coronary atherosclerotic lesions, irrespective of postmortem interval.

\section{Discussion}

One of the first literature reviews pertaining to cholesterol, triglyceride, and apolipoprotein stability in postmortem serum samples was provided by Coe $[14,15]$, who reported the results of the studies performed by Naumann [16], Glanville [17], Enticknap [18-20], Fekete and Brunsdon [21], Sturner [22], Leadbeatter and Stansbie [23], and Särkioja [12].

The results of these studies provided contradictory findings concerning cholesterol, triglyceride, and apolipoprotein (A-I and B) stability in postmortem samples and hence postmortem determination reliability. In particular, postmortem serum triglyceride levels were characterized by significant fluctuations and appeared to be of very limited value as indicators of antemortem levels [12, 14-23].

In his papers, Coe $[14,15]$ emphasized the difficulties in interpreting the postmortem values of any blood lipids due to collection of postmortem samples from individuals who were not fasting at the time of death. According to Coe [14, 15], elevated levels of these molecules measured after death should have been considered suitable exclusively in those cases characterized by an empty stomach and small intestine at autopsy. In addition, full or empty gastrointestinal tract at the time of death could have explain some of the divergent findings reported by different research teams as well as the limited success in correlating postmortem blood lipid levels with coronary artery atherosclerosis.

Three succeeding studies compared antemortem and postmortem serum lipid profiles in animals and humans, with interesting results $[10,11,24]$.

In a sample including 23 children and young adults who had been examined for cardiovascular disease risk factors and subsequently died from violent causes, Freedman et al. [10] observed that postmortem levels (postmortem serum from cardiac blood) of triglycerides and very low-density lipoprotein cholesterol were higher than antemortem values. Conversely, postmortem levels of total cholesterol and low-density and high-density lipoprotein cholesterol were related to antemortem concentrations. The strongest association between coronary artery atherosclerosis and lipid profile was observed for antemortem low-density lipoprotein cholesterol.

Hornick et al. [11] investigated cholesterol and apolipoprotein (A-I, B, E, and A-IV) concentrations in antemortem and postmortem serum samples in dogs and monkeys and failed to find appreciable differences.

Hart et al. [24] analyzed antemortem (within $72 \mathrm{~h}$ ) and postmortem (within $24 \mathrm{~h}$ of death) serum (obtained from heart or femoral blood) levels of cholesterol and triglycerides in seven autopsied hospitalized individuals and found that average postmortem cholesterol levels were $13 \%$ lower than average antemortem levels, whereas postmortem triglyceride levels were $38 \%$ higher than average antemortem triglyceride levels, thus suggesting caution in correlating postmortem lipid profiles to antemortem status.

Uemura et al. [25] measured triglyceride and total cholesterol levels in postmortem serum samples obtained from blood collected at different sampling sites (right and left heart cavities as well as femoral vein within $72 \mathrm{~h}$ postmortem). No significant differences among sampling sites were noticed in triglyceride concentrations. Conversely, total cholesterol levels showed a tendency to decrease time-dependently and lower levels in femoral vein blood more than in left cardiac blood, thus suggesting that the latter be the sample of choice to estimate antemortem cholesterol levels.

Another important line of research in the postmortem setting has explored the possible role of plasma lipids, lipoproteins, and apolipoproteins as risk factors for coronary atherosclerosis and sudden cardiac death.

Valenzuela et al. [13] observed higher levels of apolipoprotein $\mathrm{B}_{100}$ in pericardial fluid in cases with severe coronary artery atherosclerosis and positive diagnosis of myocardial infarction compared to cases without atherosclerosis.

Hiserodt et al. [26] found significantly elevated mean total cholesterol, triglycerides, low-density lipoprotein cholesterol, and apolipoprotein B concentrations in individuals who died suddenly and unexpectedly of atherosclerotic coronary artery disease compared to control cases with no significant medical or cardiac history. By contrast, mean apolipoprotein A-I and high-density lipoprotein values were not significantly different from control values, irrespective of variations in postmortem intervals or the presence of recently ingested food.

A great contribution to this topic was given by Takeishi et al. [27-32] through several investigations of postmortem plasma lipids and lipoproteins, which revealed significant correlations between remnant-like lipoprotein particle levels, coronary atherosclerosis progression (especially for cholesterol-rich particles), and sudden cardiac death incidences (especially for triglyceride-rich remnant-like particles, even in the absence of coronary atherosclerosis). These authors also observed that postmortem plasma sampled up to $12 \mathrm{~h}$ after death was appropriate for measuring lipid and lipoprotein profiles as surrogate markers of antemortem levels, including triglycerides and remnant-like lipoprotein particle-triglycerides, when an assay without added free glycerol was used. 
In addition, two significant studies deserve mentioning here. The first is that of Tsuji et al. [33], who observed that higher plasma (from right atrium blood) apolipoprotein $\mathrm{B}_{100}$ levels were associated with more severe degrees of coronary artery atherosclerosis. The second study is that of Rashid et al. [9], who observed that both total cholesterol and apolipoprotein B levels were significantly higher in cases with obstructive coronary artery disease. Furthermore, they noticed that the degree of hypercholesterolemia was positively associated with disease severity.

The results of the study presented herein tend to be in agreement with those reported in former investigations in the forensic setting. They indicate that femoral blood postmortem serum concentrations of cholesterol (total cholesterol, lowdensity lipoprotein cholesterol, and high-density lipoprotein cholesterol) and apolipoproteins (apolipoprotein B and apolipoprotein A-I) can be considered to reflect antemortem serum concentrations, thus suggesting that their postmortem values could be used as surrogates for antemortem levels. Conversely, femoral blood postmortem serum values of triglycerides do not appear to indicate antemortem concentrations accurately enough to be used as their surrogates, contrary to what was affirmed by Uemura et al. [25].

As already shown by other research teams in former reports, we found increased femoral blood postmortem serum levels of total cholesterol and apolipoprotein B in cases characterized by more severe degrees of coronary artery atherosclerosis at postmortem investigations. These results would therefore confirm that the degree of hypercholesterolemia is indeed a significant risk factor and plays a decisive role in sustaining the changes leading to vascular atherosclerotic lesions.

Lastly, our results seem to indicate that pericardial and pleural fluid lipid, lipoprotein, and apolipoprotein levels are of no value in estimating their antemortem serum concentrations, irrespective of coronary artery disease severity. If confirmed, these finding would not corroborate the data previously published by Valenzuela et al. [13].

Our study undoubtedly has some limitations. The most important is the relatively small number of studied cases, which may limit the accuracy of our research. Prospective investigations including a greater number of subjects and analyses in longer postmortem intervals would therefore be needed to confirm our findings and molecule stability.

Thus, even though further studies are required to corroborate our observations, our results seem to attest that femoral blood postmortem serum concentrations of cholesterol and apolipoproteins may be considered suitable to estimate their antemortem values in forensic cases characterized by coronary artery atherosclerotic lesions of varying severities. Conversely, to present, no alternative postmortem biological samples appear to accurately reflect the lipid/lipoprotein/apolipoprotein profile enough to be used to estimate antemortem concentrations of these compounds.
Funding This study was not financially supported.

\section{Compliance with ethical standards}

Conflict of interest The authors declare that they have no conflict of interest.

\section{References}

1. Miller M, Stone NJ, Ballantyne C, Bittner V, Criqui MH, Ginsberg HN, Goldberg AC, Howard WJ, Jacobson MS, Kris-Etherton PM, Lennie TA, Levi M, Mazzone T, Pennathur S, American Heart Association Clinical Lipidology, Thrombosis, and Prevention Committee of the Council on Nutrition, Physical Activity, and Metabolism, Council on Arteriosclerosis, Thrombosis and Vascular Biology, Council on Cardiovascular Nursing, Council on the Kidney in Cardiovascular Disease (2011) Triglycerides and cardiovascular disease. A scientific statement from the American Heart Association. Circulation 123(20):2292-2333

2. Patsch JR, Miesenböck G, Hopferwieser T, Mühlberger V, Knapp E, Dunn JK, Gotto AM Jr, Patsch W (1992) Relation of triglyceride metabolism and coronary artery disease. Studies in the postprandial state. Arterioscler Thromb 12(11):1336-1345

3. Harchaoui KE, Visser ME, Kastelein JJ, Stroes ES, Dallinga-Thie GM (2009) Triglycerides and cardiovascular risk. Curr Cardiol Rev 5(3):216-222

4. Ozturk IC, Killeen AA (1999) An overview of genetic factors influencing plasma lipid levels and coronary artery disease risk. Arch Pathol Lab Med 123(12):1219-1222

5. McBride P (2008) Triglycerides and risk for coronary artery disease. Curr Atheroscler Rep 10(5):386-390

6. Talayero BG, Sacks FM (2011) The role of triglycerides in atherosclerosis. Curr Cardiol Rep 13(6):544-552

7. Ramasamy I (2014) Recent advances in physiological lipoprotein metabolism. Clin Chem Lab Med 52(12):1695-1727

8. Bitzur R, Cohen H, Kamari Y, Shaish A, Harats D (2009) Triglycerides and HDL cholesterol: stars or second leads in diabetes ? Diabetes Care 32(Suppl 2):S373-S377

9. Rashid I, Klimis H, Duflou J, Sullivan D, Puranik R (2014) Utility of post-mortem lipid levels in fatal premature CAD: an autopsy study. Int J Cardiol 174(1):212-214

10. Freedman DS, Wattigney WA, Srinivasan S, Newman WP 3rd, Tracy RE, Byers T, Berenson GS (1993) The relation of atherosclerotic lesions to antemortem and postmortem lipid levels: the Bogalusa Heart Study. Atherosclerosis 104(1-2):37-46

11. Hornick CA, Baker HN, Malcom GT, Newman WP, Roheim PS, Strong JP (1988) Lipoproteins and apolipoproteins in postmortem serum. Mod Pathol 1(6):480-484

12. Särkioja T, Ylä-Herttuala S, Solakivi T, Nikkari T, Hirvonen J (1988) Stability of plasma total cholesterol, triglycerides, and apolipoproteins B and A-I during the early postmortem period. J Forensic Sci 33(6):1432-1438

13. Valenzuela A, Hougen HP, Villanueva E (1994) Lipoproteins and apolipoproteins in pericardial fluid: new postmortem markers for coronary atherosclerosis. Forensic Sci Int 66(2):81-88

14. Coe JI (1993) Postmortem chemistry update. Emphasis on forensic application. Am J Forensic Med Pathol 14(2):91-117

15. Coe JI (1977) Postmortem chemistry of blood, cerebrospinal fluid, and vitreous humor. Leg Med Annu 1976:55-92

16. Naumann HN (1956) Postmortem liver function tests. Am J Clin Pathol 26(5):495-505

17. Glanville JN (1960) Post-mortem serum cholesterol levels. Br Med $\mathrm{J}$ 2(5216):1852-1853 
18. Enticknap JB (1960) Biochemical changes in cadaver sera. J Forensic Med 7:135-146

19. Enticknap JB (1961) Lipids in cadaver sera after fatal heart attack. J Clin Pathol 14:496-499

20. Enticknap JB (1962) Fatty acid content of cadaver sera in fatal ischemic heart disease. Clin Sci 23:425-431

21. Fekete JF, Brundson DFV (1974) The use of routine laboratory tests in postmortem examinations. Can Soc Forensic Sci J 70: $238-254$

22. Sturner WQ (1971) Postmortem lipid studies: attempts to correlate with death from arteriosclerotic heart disease in the young age group. For Sci Gaz 2(1):5-7

23. Leadbeatter S, Stansbie D (1984) Postmortem diagnosis of familial hypercholesterolemia. Br Med (Clin Res Ed) 289(6459):1656

24. Hart AP, Zumwalt RE, Dasgupta A (1997) Postmortem lipid levels for the analysis of the risk factors of sudden death: usefulness of the Ektachem and Monarch analyzers. Am J Forensic Med Pathol 18(4):354-359

25. Uemura K, Shintani-Ishida K, Saka K, Nakajima M, Ikegaya H, Kikuchi Y, Yoshida K (2008) Biochemical blood markers and sampling sites in forensic autopsy. J Forensic Legal Med 15(5):312-317

26. Hiserodt JC, Perper JA, Koehler SA, Orchard TJ (1995) A comparison of blood lipid and lipoprotein values in young adults who die suddenly and unexpectedly from atherosclerotic coronary artery disease with other noncardiac deaths. Am J Forensic Med Pathol 16(2):101-106

27. Takeichi S, Nakajima Y, Osawa M, Yukawa N, Saito T, Seto Y, Nakano T, Adachi M, Jitsukata K, Horiuchi K, Wang T, Nakajima
K (1997) The possible role of remnant-like particles as a risk factor for sudden cardiac death. Int J Legal Med 110(4):213-219

28. Takeichi S, Yukawa N, Nakajima Y, Osawa M, Saito T, Seto Y, Nakano T, Saniabadi AR, Adachi M, Wang T, Nakajima K (1999) Association of plasma triglyceride-rich lipoprotein remnants with coronary atherosclerosis in cases of sudden cardiac death. Atherosclerosis 142(2):309-315

29. Takeichi S, Nakajima Y, Yukawa N, Saito T, Seto Y, Huang XL, Kusakabe T, Jin ZB, Hasegawa I, Nakano T, Saniabadi A, Adachi M, Ohara N, Wang T, Nakajima K (2001) Plasma triglyceride-rich lipoprotein remnants as a risk factor of 'Pokkuri disease'. Leg Med (Tokyo) 3(2):84-94

30. Takeichi S, Nakajima Y, Yukawa N, Fujita MQ, Saito T, Satoh F, Seto Y, Kusakabe T, Jin ZB, Hasegawa I, Nakano T, Saniabadi A, Adachi M, Ohara N, Usui S, Okazaki M, Nakajima K (2004) Validity of plasma remnant lipoproteins as surrogate markers of antemortem level in cases of sudden coronary death. Clin Chim Acta 343(1-2):93-103

31. Nakajima Y, Takeichi S, Osawa M, Nakajima K, Nakano T, Fujita MQ (2006) The lipid and lipoprotein profiles of apo B-carrying particles in remnant-like lipoproteins isolated from plasma in sudden cardiac death cases. Tokai J Exp Clin Med 31(4):160-166

32. Nakajima K, Nakajima Y, Takeichi S, Fujita MQ (2007) ApoB-100 carrying lipoprotein, but not apoB-48, is the major subset of proatherogenic remnant-like lipoprotein particles detected in plasma of sudden cardiac death cases. Atherosclerosis 194(2):473-482

33. Tsuji A, Ikeda N, Nakamura T (1999) Plasma lipids, lipoproteins and apolipoproteins and sudden cardiac death. Int $\mathrm{J}$ Legal Med 112(3):151-154 